\title{
Redes de colaboración y producción científica sudamericana en medicina clínica, ISI Current Contents 2000-2009
}

'Universidad Nacional

Mayor de San Marcos,

Sociedad Científica de San Fernando. Lima, Perú.

${ }^{2}$ Universitat de València, Departamento de

Historia de la Ciencia y Documentación. Valencia, España. ${ }^{3}$ Universidad Peruana Cayetano Heredia. Lima, Perú.

${ }^{4}$ Universidad Nacional Mayor de San Marcos, Facultad de Medicina. Lima, Perú.

Este trabajo no recibió financiamiento externo Conflictos de Interés: Ninguno

Recibido el 17 de agosto de 2011, aceptado el 21 de diciembre de 2011.

Correspondencia a: Charles Huamaní Saldaña Av. Arriba Perú 1154, Villa el Salvador. Lima 42, Perú Teléfono: (+51-1) 9928$14710 / 287-1397$ E-mail: huamani_ca@ hotmail.com

\author{
CHARLES HUAMANI ${ }^{1}$, GREGORIO GONZÁLEZ A. ${ }^{2}$, \\ WALTER H. CURIOSO ${ }^{3}$, JOSÉ PACHECO-ROMERO ${ }^{4}$

\section{Scientific production in clinical medicine and international collaboration networks in South American countries}

Background: International collaboration is increasingly used in biomedical research. Aim: To describe the characteristics of scientific production in Latin America and the main international collaboration networks for the period 2000 to 2009. Material and Methods: Search for papers generated in Latin American countries in the Clinical Medicine database of ISI Web of Knowledge v.4.10 - Current Contents Connect. The country of origin of the corresponding author was considered the producing country of the paper. International collaboration was analyzed calculating the number of countries that contributed to the generation of a particular paper. Collaboration networks were graphed to determine the centrality of each network. Results: Twelve Latin American countries participated in the production of 253,362 papers. The corresponding author was South American in 79\% of these papers. Sixteen percent of papers were on clinical medicine and $36 \%$ of these were carried out in collaboration. Brazil had the highest production (22,442 papers) and the lower percentage of international collaboration (31\%). North America accounts for 63\% of collaborating countries. Only 8\% of collaboration is between South American countries. Brazil has the highest tendency to collaborate with other South American countries. Conclusions: Brazil is the South American country with the highest scientific production and indicators of centrality in South America. The most common collaboration networks are with North American countries.

(Rev Med Chile 2012; 140: 466-475).

Key words: Biomedical research; Clinical medicine; Publications; South America.
$\mathrm{E}$ lincremento de la colaboración internacional es una característica de la evolución de la ciencia en las últimas décadas ${ }^{1,2}$, ello está justificado por el carácter multidisciplinar y especializado de las investigaciones; por la necesidad de acceder a tecnologías y equipos más complejos, sofisticados y caros, y por los beneficios obtenidos en términos de citación e impacto ${ }^{3,4}$. Varios estudios señalan una relación positiva entre la colaboración internacional y las investigaciones de mayor calidad y relevancia científica, medido a través del grado de citación ${ }^{5,6}$.
En otras áreas existen diversos organismos o programas que fomentan la colaboración internacional. Por ejemplo, el 'Programa Iberoamericano de Cooperación en Ciencia y Tecnología para el Desarrollo' (CYTED) que incluye el área de salud, promueve la inclusión científica y tecnológica ${ }^{7}$; y en informática biomédica, la red QUIPU ${ }^{8}$ promueve la colaboración en educación e investigación.

Para fomentar la colaboración se dispone de varios mecanismos como el intercambio de investigadores, cursos, financiamiento, entre otros 9 . Sin embargo, los resultados de esta cooperación se 
miden valorando la colaboración en publicaciones ${ }^{10}$ y sus características ${ }^{11}$.

En Sudamérica, se han realizado estudios bibliométicos en todas las ciencias, mostrando la cuantificación parcial o absoluta de los trabajos en cooperación ${ }^{12,13}$, donde la colaboración internacional ha sido muy variable, por ejemplo, entre 1991 y 1995 los artículos en colaboración internacional con participación de Argentina eran de $23 \%$, mientras que para Bolivia era de $70 \%{ }^{12}$.

Aunque en estos estudios se brindan indicadores que señalan la colaboración, no se complementan con representaciones gráficas de las redes de colaboración, además podemos obtener otros indicadores que midan con mayor precisión la colaboración científica ${ }^{14}$, el grado de internacionalización y la orientación de la cooperación en la producción de los diferentes países. Las redes de colaboración entre países se pueden constituir entre aquellos que guardan nexos históricos y geográficos, o más formalmente, si los países entablan relaciones diplomáticas para su integración, como sucede en Sudamérica a través de la constitución de la Unión de Naciones Sudamericanas (UNASUR).

El objetivo de este trabajo es describir las características de la producción científica sudamericana en medicina clínica entre los años 2000 y 2009, además de conocer las principales redes de colaboración; pretendiendo ofrecer una visión actualizada de los estudios métricos que analizan la productividad y la colaboración científica.

\section{Materiales y Métodos}

\section{A. Búsquedas documentales y procesamiento de la información}

Se realizó un estudio descriptivo, iniciando con diferentes búsquedas documentales en la base de datos ISI Web of Knowledge v.4.10 - Current Contents Connect; a la cual se accedió a través del sistema de bibliotecas de la Universidad Nacional Mayor de San Marcos. La búsqueda incluyó al sector sudamericano de la región de América Latina y el Caribe, siguiendo la clasificación de las Naciones Unidas (doce países: Argentina, Bolivia, Brasil, Chile, Colombia, Ecuador, Guyana, Paraguay, Perú, Surinam, Uruguay y Venezuela) y los años de publicación 2000 a 2009. Además, como criterio de búsqueda seleccionamos la base de datos Clinical
Medicine, por ser la que reúne la mayor cantidad de revistas en medicina (1.478 revistas en mayo de 2010). Se excluyeron los libros o secciones de libros, quedando sólo las publicaciones en revistas científicas (Journals); estos registros fueron procesados considerando las siguientes definiciones:

- Documento y artículo: las publicaciones recuperadas podían ser artículos, revisiones, casos clínicos, entre otras; que en conjunto llamaremos 'documentos', y serán empleados para indicadores generales de producción; mientras que los 'artículos', que son resultados de investigaciones, serán analizados por separado.

- Firma país: es el país al que pertenece la institución declarada por el autor. Consideramos las firmas país distintas por artículo. Los análisis efectuados se basaron en la cuantificación total y parcial de las firmas país.

- País productor y país colaborador: se considera como país productor al país del autor corresponsal y como colaborador a las demás firmas de países que figuraban en el artículo. Priorizamos al autor corresponsal porque en las publicaciones biomédicas el orden de las firmas se percibe de diferente manera. Así, se otorga un mayor peso a la primera y última posición $^{15-16}$, asociadas generalmente con los autores corresponsales, confiriéndoles un papel de liderazgo en la ejecución, dirección o supervisión de las investigaciones ${ }^{17}$.

Para los artículos se generó una hoja Excel por cada país sudamericano donde este actuaba como productor, y otra para todos los países no sudamericanos. Codificamos cada artículo conservando las firmas de países; clasificando los países por regiones.

\section{B. Determinación de indicadores bibliométricos}

Calculamos en Excel los siguientes indicadores de actividad científica para todos los países sudamericanos, aplicando estadística descriptiva (frecuencias y porcentajes) y representaciones gráficas:

- Documentos en ISI Current Contents (todas las bases de datos) correspondientes a cada país sudamericano y su relación con la producción total.

- Documentos en la base de datos de medicina clínica y porcentajes relativos por cada país, indicando el porcentaje donde el autor corresponsal era del país evaluado. 
- Índices de producción ISI total y en medicina clínica por millón de habitantes y por Producto Bruto Interno (PBI); que permiten relativizar la actividad científica de los países analizados considerando sus diferentes tamaños poblacionales y desarrollo económico. Estos datos se obtuvieron de la página electrónica de CIA World Factbook (https://www.cia.gov/library/ publications/the-world-factbook/), con información actualizada a junio de 2009.

- Número y porcentaje de artículos correspondientes a la producción científica en medicina clínica, con autor corresponsal del país evaluado, y en colaboración internacional.

- Colaboración de Sudamérica con otras regiones del mundo.

\section{Análisis de redes de colaboración}

Para graficar la colaboración sudamericana se construyeron dos redes de colaboración usando las firmas país generadas:

- En la primera red se incluyó a doce países sudamericanos y a 18 países de regiones que más colaboraron.

- En la segunda red se mostró sólo las colaboraciones entre países sudamericanos.

Para realizar el análisis gráfico, utilizamos el programa de representación visual Pajek $^{\circledast}$ v.2.04 (http://pajek.imfm.si), con el que se obtuvo, además, tres indicadores que permitieron evaluar la relación de cada país con la red de colaboración ${ }^{14}$, estos son:

- Grado: indica el número de países con los que un país ha colaborado; es una medida que refleja el grado de vinculación internacional.

- Intermediación: evalúa en qué medida un país estaba 'en medio' o permitía la interconexión entre otros miembros de la red; por tanto, mide el acceso y control de los flujos de información.

- Cercanía: refleja la 'proximidad' de cada país con el resto de los miembros que integran la red; valorando la rapidez de interacción de un país con otro.

\section{Resultados}

Entre 2000 y 2009, los doce países sudamericanos participaron en la producción de 253.362 documentos en revistas de ISI Current Contents, siendo Brasil el país con mayor producción, equivalente al 57,6\% del total sudamericano. Bolivia, Paraguay, Guyana y Surinam contribuyen en conjunto con menos de 1\%. El nivel de producción total por país es similar a la observada al cuantificar la producción en medicina clínica (Tabla 1); que representa el 15,8\% del total de la producción; aunque para Perú, Paraguay y Surinam representa más de $30 \%$ de su producción.

De la producción en medicina clínica evaluada, el 73,7\% corresponde a documentos cuyo corresponsal es de un país sudamericano; siendo muy variable, pues Brasil es corresponsal de $81,1 \%$ de los documentos donde participó; mientras que siete países son corresponsales de menos de 50\% del total de documentos en los que participaron.

El análisis de la producción difiere si se usan indicadores socioeconómicos. Así, el orden según PBI ubica a Chile en primer lugar, seguido de Uruguay, Argentina y Brasil como los que más documentos tienen según PBI. Este patrón es similar al que se obtiene con el número de documentos por millón de habitantes. Al realizar este ordenamiento, Colombia y Venezuela descienden a las últimas posiciones en el índice documentos en medicina clínica/PBI, y mantienen posiciones intermedias en los demás (Tabla 2).

El número de documentos publicados el 2009 fue $47,5 \%$ más que los publicados el 2000, con un promedio de crecimiento anual de 6,9\% (Figura 1). Bolivia experimentó un crecimiento de 4,1 veces su producción, seguido de Brasil que incrementó en 3,5 veces.

Del total de documentos en medicina clínica, el $82,7 \%$ correspondió a artículos; de estos, se excluyeron $139(0,3 \%)$ por una presentación inadecuada de datos y $33(0,1 \%)$ porque no se pudo determinar el país productor.

De los incluidos $(\mathrm{n}=34.152)$, los artículos en colaboración internacional representan el 36,3\% (12.397 artículos) de la producción sudamericana, apreciando un incremento anual en las colaboraciones al pasar de 35,3\% el año 2000 al 38,0\% en 2009. Al calcular el porcentaje de artículos en colaboración por países, la mayoría incrementó su producción en el período 2005-2009 comparado al 2000-2004, siendo mayor para Venezuela (16,0\%) y Ecuador (15,1\%). De otro lado, disminuyó el número de artículos siendo corresponsal en el caso de Venezuela $(10,7 \%)$ y Uruguay $(11,3 \%)$. El país con mayor producción de artículos como 
Tabla 1. Producción científica sudamericana en ISI Current Contents: 2000 a 2009

\begin{tabular}{|c|c|c|c|c|c|c|}
\hline \multirow[b]{2}{*}{ Brasil } & \multicolumn{2}{|c|}{ Total de documentos } & \multicolumn{2}{|c|}{$\begin{array}{l}\text { Documentos en Medicina } \\
\text { clínica* }\end{array}$} & \multicolumn{2}{|c|}{$\begin{array}{l}\text { Documentos en medicina } \\
\text { clínica siendo corresponsal }\end{array}$} \\
\hline & 152.014 & $(57,6 \%)$ & 26.769 & $(17,6 \%)$ & 21.720 & $(81,1 \%)$ \\
\hline Argentina & 52.190 & $(19,8 \%)$ & 5.826 & $(11,2 \%)$ & 3.905 & $(67,0 \%)$ \\
\hline Chile & 28.666 & $(10,9 \%)$ & 4.814 & $(16,8 \%)$ & 3.657 & $(76,0 \%)$ \\
\hline Venezuela & 9.889 & $(3,7 \%)$ & 1.330 & $(13,4 \%)$ & 819 & $(48,1 \%)$ \\
\hline Colombia & 9.609 & $(3,6 \%)$ & 1.701 & $(17,7 \%)$ & 696 & $(52,3 \%)$ \\
\hline Perú & 3.723 & $(1,4 \%)$ & 1.215 & $(32,6 \%)$ & 375 & $(30,9 \%)$ \\
\hline Uruguay & 4.178 & $(1,6 \%)$ & 637 & $(15,2 \%)$ & 346 & $(54,3 \%)$ \\
\hline Ecuador & 1.888 & $(0,7 \%)$ & 380 & $(20,1 \%)$ & 143 & $(37,6 \%)$ \\
\hline Bolivia & 1.291 & $(0,5 \%)$ & 230 & $(17,8 \%)$ & 52 & $(22,6 \%)$ \\
\hline Paraguay & 304 & $(0,1 \%)$ & 109 & $(35,9 \%)$ & 26 & $(23,9 \%)$ \\
\hline Guyana & 147 & $(<0,1 \%)$ & 35 & $(23,8 \%)$ & 12 & $(34,3 \%)$ \\
\hline Surinam & 76 & $(<0,1 \%)$ & 25 & $(32,9 \%)$ & 4 & $(16,0 \%)$ \\
\hline Total $^{\ddagger}$ & 253.362 & (100\%) & 40.069 & $(15,8 \%)$ & 31.755 & $(79,3 \%)$ \\
\hline
\end{tabular}

Base de datos extraída en abril de 2010. *Porcentaje que aporta la colección Clinical Medicine. †Documentos publicados en los

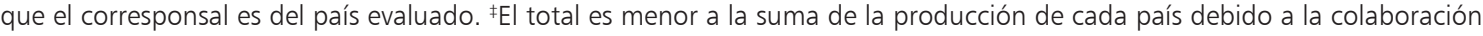
sudamericana existente.

Tabla 2. Índices de producción científica* sudamericana en revistas recogidas de ISI Current Contents según habitantes y PBI para el 2009

\begin{tabular}{|c|c|c|c|c|c|c|}
\hline & \multirow[t]{2}{*}{$\mathbf{P B I}^{\dagger}$} & \multirow[t]{2}{*}{$\begin{array}{l}\text { Población } \\
\text { estimada }^{\dagger}\end{array}$} & \multicolumn{2}{|c|}{ Índice producción total } & \multicolumn{2}{|c|}{$\begin{array}{c}\text { Índice producción en medicina } \\
\text { clínica }\end{array}$} \\
\hline & & & $\mathbf{x}$ PBI & x 1.000.000 hab. & x PBI & x 1.000.000 hab. \\
\hline Brasil & $1.794,0$ & 198.739 .269 & 12,1 & 108,8 & 2,4 & 22,0 \\
\hline Argentina & 494,3 & 40.913 .584 & 12,8 & 154,2 & 1,5 & 17,6 \\
\hline Colombia & 358,9 & 45.644 .023 & 2,8 & 22,0 & 0,7 & 5,3 \\
\hline Venezuela & 314,6 & 26.814 .843 & 4,8 & 56,8 & 0,5 & 5,4 \\
\hline Chile & 226,3 & 16.601 .707 & 17,0 & 231,8 & 2,7 & 36,5 \\
\hline Perú & 206,8 & 29.546 .963 & 2,7 & 19,0 & 1,0 & 6,7 \\
\hline Ecuador & 98,7 & 14.573 .101 & 3,1 & 21,0 & 0,6 & 3,8 \\
\hline Bolivia & 38,9 & 9.775 .246 & 4,9 & 19,5 & 0,9 & 3,4 \\
\hline Uruguay & 36,9 & 3.494 .382 & 15,1 & 159,1 & 2,3 & 24,0 \\
\hline Paraguay & 25,6 & 6.995 .655 & 1,3 & 4,9 & 0,6 & 2,3 \\
\hline Guyana & 2,7 & 772.298 & 4,9 & 17,5 & 1,3 & 4,5 \\
\hline Surinam & 3,8 & 481.267 & 1,4 & 11,4 & 0,5 & 4,2 \\
\hline Total & -- & 394.352 .338 & -- & 91,2 & -- & 21,5 \\
\hline
\end{tabular}

*Índice de Producción: (Documentos publicados el 2008 y 2009) / 1.000.000 hab. (habitantes) 2009 o PBI (Producto Bruto

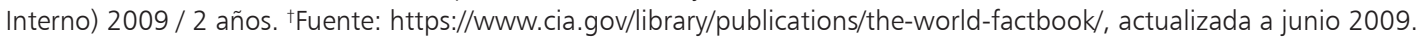




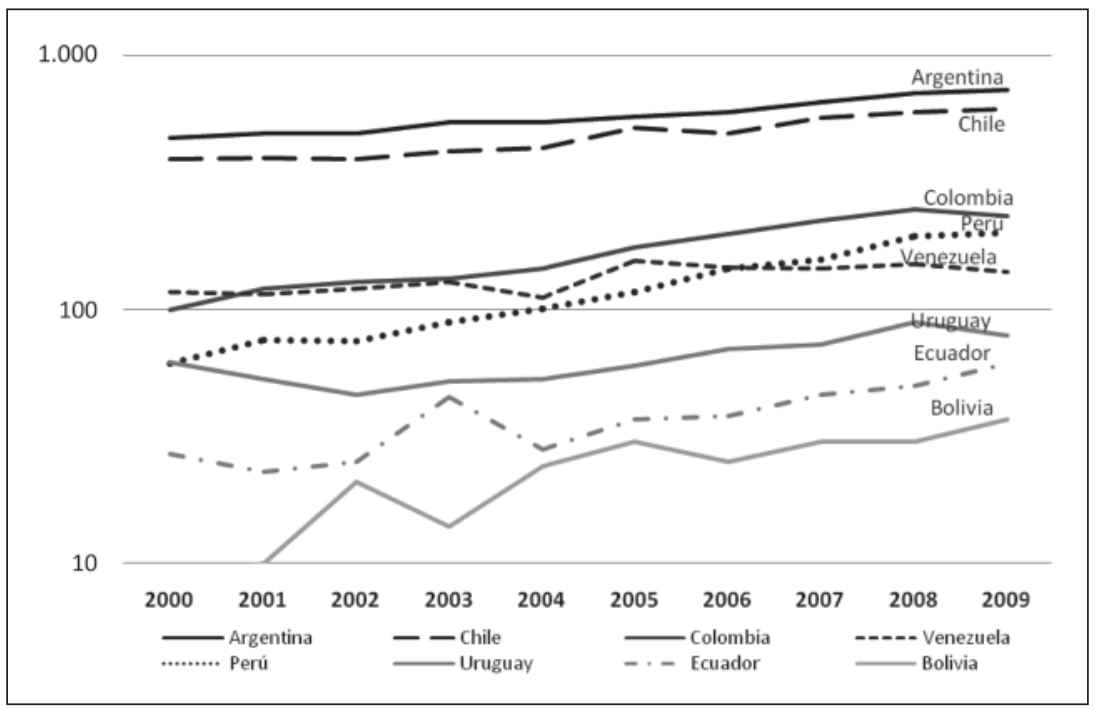

Figura 1. Evolución anual de las publicaciones sudamericanas en revistas en ISI Current Contents [Clinical Medicine]. 2000 a 2009*. *Escala logarítmica. Para facilitar la interpretación de la imagen, no mostramos la producción de Brasil (que supera los 1.000 artículos al año), ni de Guyana ni Surinam. corresponsal fue Brasil; Bolivia fue el país con mayor porcentaje de artículos en colaboración internacional (Tabla 3).

Con relación al número de países en un artículo, predomina la colaboración internacional entre dos países $(65,9 \%$ de los artículos en cola- boración), frente a las colaboraciones entre tres $(16,8 \%)$ o cuatro países $(5,2 \%)$; con una tendencia al incremento del número de países que firman los artículos en colaboración, registrando incluso colaboraciones de más de 20 países (Figura 2).

De los 12.397 artículos realizados en colabora-

Tabla 3. Colaboración en artículos con participación sudamericana en revistas en ISI Current Contents [Clinical Medicine], entre 2000-2009

\begin{tabular}{|c|c|c|c|c|c|c|c|}
\hline \multirow{2}{*}{$\begin{array}{l}\text { País } \\
\text { 1. Brasil }\end{array}$} & \multirow{2}{*}{$\begin{array}{c}\text { Total de } \\
\text { artículos } \\
\text { originales (A) } \\
22.442\end{array}$} & \multicolumn{2}{|c|}{$\begin{array}{l}\text { Artículos como } \\
\text { corresponsal (B) }\end{array}$} & \multicolumn{2}{|c|}{$\begin{array}{c}\text { Artículos en } \\
\text { colaboración } \\
\text { internacional }(C)^{2}\end{array}$} & \multicolumn{2}{|c|}{$\begin{array}{l}\text { Artículos en colaboración } \\
\text { internacional siendo } \\
\text { corresponsal }(B \cap C)^{3}\end{array}$} \\
\hline & & 18.228 & $(81,2 \%)$ & 6.991 & $(31,2 \%)$ & 2.819 & $(40,3 \%)$ \\
\hline 2. Argentina & 4.666 & 3.020 & $(64,7 \%)$ & 2.210 & $(47,4 \%)$ & 591 & $(26,7 \%)$ \\
\hline 3. Chile & 3.887 & 3.002 & $(77,2 \%)$ & 1.347 & $(34,7 \%)$ & 468 & $(34,7 \%)$ \\
\hline 4. Colombia & 1.367 & 609 & $(44,6 \%)$ & 961 & $(70,3 \%)$ & 217 & $(22,6 \%)$ \\
\hline 5. Venezuela & 1.005 & 486 & $(48,4 \%)$ & 590 & $(58,7 \%)$ & 136 & $(23,1 \%)$ \\
\hline 6. Perú & 987 & 250 & $(25,3 \%)$ & 872 & $(88,3 \%)$ & 143 & $(16,4 \%)$ \\
\hline 7. Uruguay & 490 & 262 & $(53,5 \%)$ & 331 & $(67,6 \%)$ & 107 & $(32,3 \%)$ \\
\hline 8. Ecuador & 294 & 99 & $(33,7 \%)$ & 234 & $(79,6 \%)$ & 43 & $(18,4 \%)$ \\
\hline 9. Bolivia & 198 & 40 & $(20,2 \%)$ & 184 & $(92,9 \%)$ & 24 & $(13,0 \%)$ \\
\hline 10. Paraguay & 93 & 21 & $(22,6 \%)$ & 85 & $(91,4 \%)$ & 13 & $(15,3 \%)$ \\
\hline 11. Surinam & 23 & 4 & $(17,4 \%)$ & 20 & $(87,0 \%)$ & 1 & $(5,0 \%)$ \\
\hline 12. Guyana & 24 & 5 & $(20,8 \%)$ & 20 & $(83,3 \%)$ & 1 & $(5,0 \%)$ \\
\hline Total* & 34.152 & 26.026 & $(76,2 \%)$ & 12.397 & $(36,3 \%)$ & 4.563 & $(36,8 \%)$ \\
\hline
\end{tabular}

Porcentajes: ${ }^{1}(\mathrm{~B} / \mathrm{A}) ;{ }^{2}(\mathrm{C} / \mathrm{A}) ;{ }^{3}(\mathrm{~B} \cap \mathrm{C}) / \mathrm{C}$. *El total difiere de la suma pues existen artículos en colaboración donde participó más de un país sudamericano. 


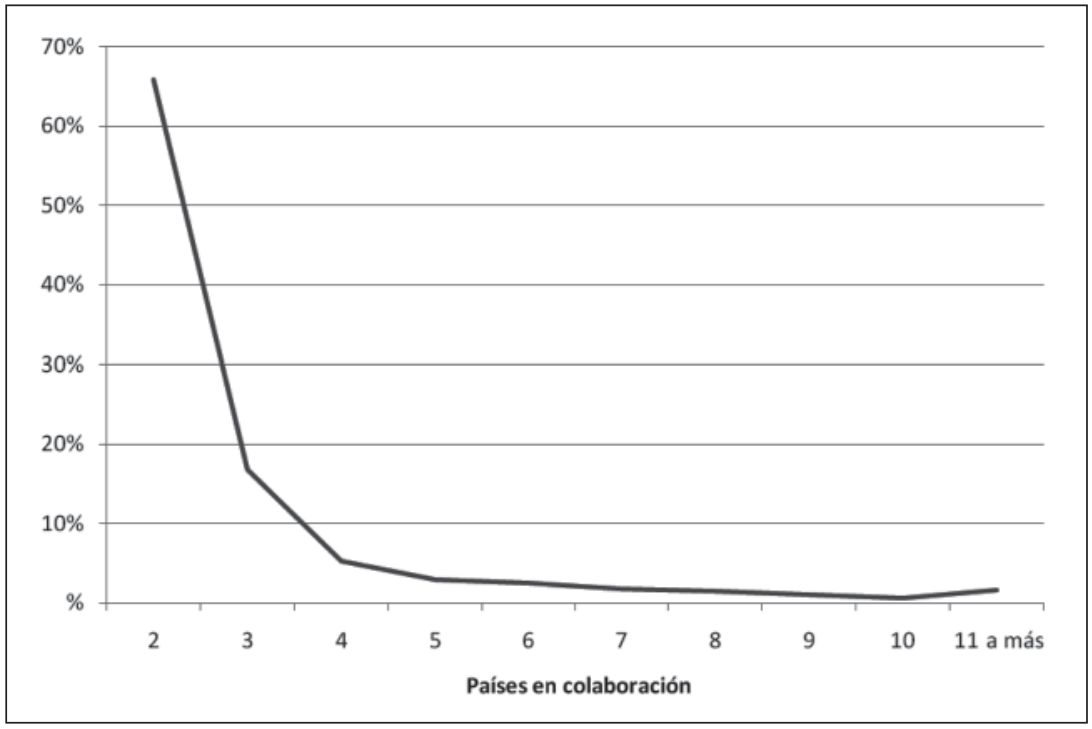

Figura 2. Porcentaje de países en colaboración en artículos con participación sudamericana en revistas en ISI Current Contents [Clinical Medicine], entre 20002009 ción, 1.060 (3,1\%) artículos tienen colaboración entre países sudamericanos y $12.084(35,4 \%)$ artículos la tienen con países de otras regiones. De estos, Norteamérica colaboró en el 64,9\% de los artículos firmados por al menos un país sudamericano y, Europa en el 50,1\% (Tabla 4); la colaboración europea se incrementó, pues pasó de $46,8 \%$ artículos en colaboración en 2000-2004 al 51,9\% en 2005-2009 (datos no presentados en tablas).

Los países no sudamericanos que más colaboraron fueron Estados Unidos de Norteamérica (EEUU), Reino Unido y Francia. De los países sudamericanos, Brasil colaboró con 137 países y Argentina con 116; el ordenamiento del grado de colaboración es similar al del número de publicaciones (Tabla 5). Se observó la participación de 155 países en todo el período.

Si bien los países con mayor producción son las principales 'intermediarios' con otros países o los de mayor 'proximidad' en las redes de colaboración; también destaca la presencia de algunos países con una producción menor, que ocupan buenas posiciones como 'intermediarios' o en razón de su 'proximidad'.
En el período 2000-2009, la colaboración internacional incrementó en intensidad pero sin muchos cambios en su distribución; Brasil y Argentina son los paises sudamericanos que tienen mayor número de colaboraciones (Figura 3). En el mapa de colaboración se aprecia que el centro de la red está conformado por Brasil, Argentina, EEUU y varios países europeos (Reino Unido, Francia, Italia entre otros), siendo

Tabla 4. Colaboración en artículos con participación sudamericana en revistas en ISI Current Contents [Clinical Medicine] según regiones, 2000-2009

\begin{tabular}{|lcc|}
\hline Región & $\begin{array}{c}\text { n de artículos } \\
\text { en colaboración }\end{array}$ & $\begin{array}{c}\text { \% respecto al } \\
\text { total de artículos } \\
\text { en colaboración }\end{array}$ \\
\hline Norte América & 7.846 & 63,3 \\
\hline Europa & 6.056 & 48,8 \\
\hline Asia & 1.325 & 10,7 \\
\hline Sudamérica* & 1.060 & 8,5 \\
\hline Centro América y el Caribe & 962 & 7,8 \\
\hline Oceanía & 640 & 5,2 \\
\hline África & 430 & 3,5 \\
\hline Total & $12.397^{\dagger}$ & $36,3^{\dagger}$ \\
\hline
\end{tabular}

* Documentos en colaboración intrasudamericana de dos o más países. ${ }^{\dagger}$ Aunque las colaboraciones se realizaron en 12.397 artículos diferentes hay que tener presente que en algunos artículos concurren colaboraciones simultáneas intrasudamericanas o con países de diferentes regiones geográficas de ahí que los sumatorios de las columnas no se corresponda con el valor expresado en el total. 
Tabla 5. Indicadores de centralidad del Análisis de Redes Sociales determinados a partir de las colaboraciones en artículos con participación sudamericana en revistas en ISI Current Contents [Clinical Medicine], 2000-2009

\begin{tabular}{|lclclc|}
\hline País & Grado & País & $\begin{array}{c}\text { Intermediación } \\
(\mathbf{x ~ 1 0 0 )}\end{array}$ & País & $\begin{array}{c}\text { Cercanía } \\
(\mathbf{x ~ 1 0 0 )}\end{array}$ \\
\hline Brasil & 137 & Brasil & 9,54 & Brasil & 90,06 \\
\hline Argentina & 116 & Argentina & 2,55 & Argentina & 80,21 \\
\hline Chile & 101 & Chile & 2,08 & Chile & 74,40 \\
\hline Perú & 96 & Perú & 1,50 & Perú & 72,64 \\
\hline Colombia & 96 & Bolivia & 1,16 & Colombia & 72,64 \\
Venezuela & 78 & Colombia & 1,11 & Venezuela & 66,96 \\
\hline Ecuador & 71 & Ecuador & 0,81 & Ecuador & 64,98 \\
\hline Uruguay & 69 & Venezuela & 0,61 & Uruguay & 64,44 \\
\hline Bolivia & 62 & Uruguay & 0,24 & Bolivia & 62,60 \\
\hline Paraguay & 41 & Surinam & 0,20 & Paraguay & 57,68 \\
\hline Surinam & 15 & Paraguay & 0,14 & Surinam & 52,20 \\
Guyana & 10 & Guyana & 0,07 & Guyana & 51,16 \\
\hline
\end{tabular}

Grado: es el número de países diferentes con las que ha colaborado; refleja el tamaño del círculo de colaboradores del país. Intermediación: evalúa la capacidad de acceder y controlar los flujos de información. Cercanía: valora la proximidad al resto de países que conforman la red.

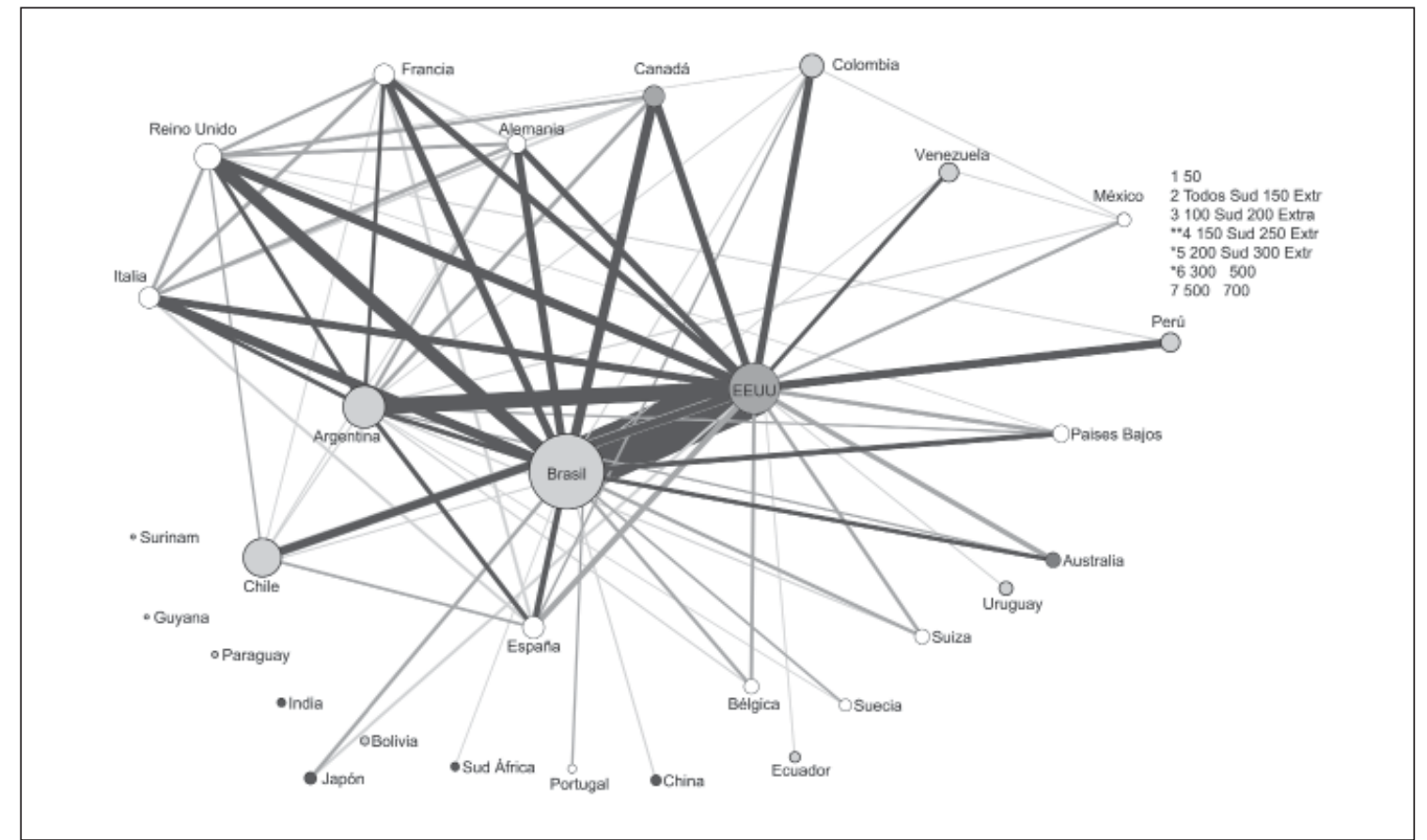

Figura 3. Mapa de colaboración en artículos con participación sudamericana, publicados en revistas en ISI Current Contents [Clinical Medicine] entre 2000-2009. Se grafican los 12 países sudamericanos y a los 18 países que más colaboran con ellos en la producción de artículos originales. El grosor y color de las líneas es proporcional al número de artículos en colaboración, y el tamaño de los nodos es proporcional al número de artículos publicados por los países sudamericanos y al número de trabajos en colaboración con los mismos en el caso del resto de países. En gris oscuro se grafican las colaboraciones Sur-sur de más de 200 artículos y Sur-otros países de más de 300. 


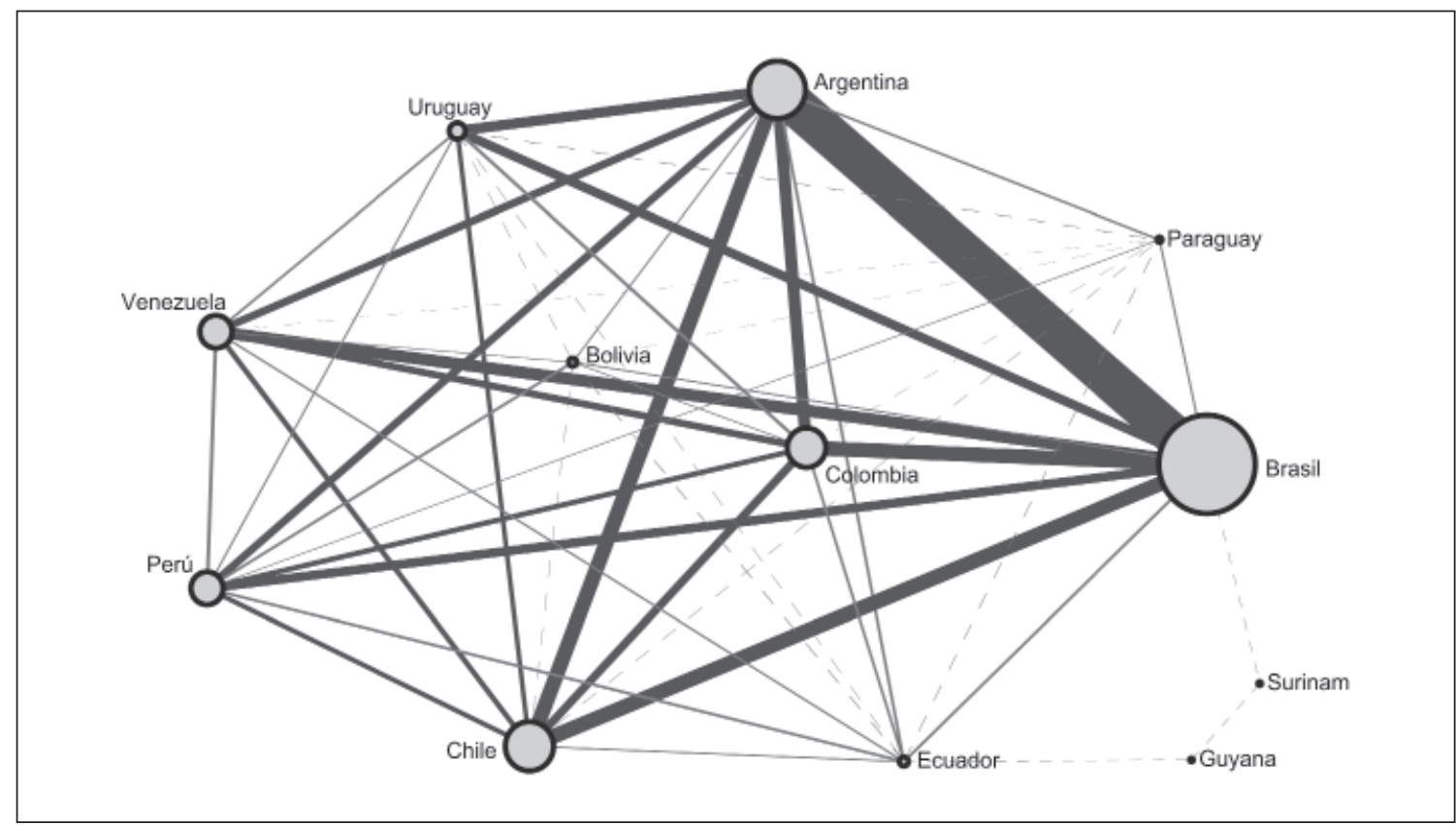

Figura 4. Mapa de colaboración sudamericana en artículos publicados en revistas en ISI Current Contents [Clinical Medicine] entre 2000-2009. Se grafican todas las colaboraciones entre países sudamericanos. El grosor de las líneas es proporcional al número de artículos en colaboración, y el tamaño de los nodos al número de artículos publicados por dicho país.

EEUU y Brasil los intermediarios de la colaboración de los demás paises sudamericanos. En el período 2000-2009 la máxima colaboración fue la de Brasil-EEUU con 4.056 artículos.

Al ver la colaboración entre países sudamericanos (Figura 4) se aprecia que, salvo Guyana y Surinam, los demás países interactúan. En la mayoría de los casos, las colaboraciones generadas por un país con los demás, son de similar intensidad y proporcionales a su producción; aunque existe una mayor colaboración con Argentina y Brasil, en especial de Uruguay y Chile.

\section{Discusión}

Brasil es el país con mayor producción científica $(57,7 \%)$ de 2000 a 2009 . Esto concuerda con estudios previos donde se encuentra que Brasil publicó el 48,2\% de documentos sudamericanos entre $1991-1995^{12}$ y $54,9 \%$ entre $1999-2002^{13}$; esto podría sugerir que la producción científica de Brasil está en aumento, lo cual requiere estudios posteriores.

Aunque son varios los factores que intervie- nen en el incremento de la producción científica de un país, como inversión o personal dedicado, nuestro estudio sugiere que los países necesitan de la colaboración internacional para incrementar su producción, principalmente de EEUU y países europeos.

También es importante destacar que Chile, Uruguay y Argentina, presentan mejores índices de producción cuando ajustamos los resultados según el PBI o el número de habitantes. Aunque nuestros resultados difieren mucho referente a lo informado para salud el $2007^{9}$, posiblemente por la metodología empleada para cuantificar las publicaciones, coincide en que Uruguay es el país que resalta en estos índices. Esto puede explicarse porque su población es principalmente urbana y conglomera aproximadamente el $85 \%$ en su capital. Mientras que otros países tienen una población más distribuida y pocas ciudades con producción científica. Como ejemplo, en Perú, su capital, Lima, aporta el $95 \%$ de sus publicaciones ${ }^{19}$, pero conglomera menos de $30 \%$ de la población del país.

En cuanto a la colaboración internacional, la elección de los países colaboradores está determinada por diversos factores ${ }^{3}$, destacando la inclinación 
hacia colaborar con países con mayor desarrollo científico ${ }^{5}$. Así, a mayor desarrollo científico la colaboración será mayor, pero su necesidad disminuye. Esta situación explicaría por qué la colaboración internacional en investigación es inversamente proporcional al tamaño científico del país ${ }^{20,21}$. Este aspecto se sugiere en nuestro trabajo al observar que los países de menor productividad científica presentan una mayor tendencia a la colaboración.

Brasil y Argentina tienen la mayor producción y menor porcentaje de colaboración; pero también tienen las colaboraciones más intensas con países no sudamericanos, conservando un porcentaje importante de autorías corresponsales. Esto refleja la autonomía que han obtenido estos países en medicina $^{22}$, lo que les permite interactuar más y mejor en la red, siendo intermediarios de otros países y facilitando el incremento de su producción.

Otro factor que favorece la colaboración internacional es la existencia de vínculos históricos, culturales, idiomáticos, científicos o geográficos ${ }^{23,24}$. En Sudamérica, estos factores parecen no tener influencia, pues incluso es menor que la colaboración con Asia; igualmente, España, Portugal u otros países de Centroamérica, con quienes se tiene importantes vínculos, tienen un papel por detrás de EEUU u otros países europeos. Una posible explicación es que al colaborar con países de otras regiones, en especial con EEUU, se priorizaría la publicación en revistas de habla inglesa, que tienen un mayor factor de impacto ${ }^{11,25}$ y están mejor representadas en ISI Current Contents ${ }^{26}$.

Al analizar la colaboración entre sudamericanos se aprecia una preferencia por Argentina o Brasil, en lugar de otros países vecinos. Aunque esta colaboración ha incrementado, aún es menor que con países no sudamericanos, lo que sugiere que se necesitan reforzar los programas de cooperación Sur-Sur'. En la actualidad, los sistemas nacionales de muchos países en Sudamérica están en desarrollo y los acuerdos son preliminares ${ }^{9,22}$; en ese sentido, se deben establecer mayores convenios de cooperación y fomentar los sistemas nacionales de investigación en salud, apoyado por un marco legal adecuado y políticas que prioricen la investigación.

El trabajo tiene algunas limitaciones. Primero, no hubo correlación entre la dirección del autor corresponsal y el país firmante en $0,4 \%$ de los artículos, que fueron excluidos; segundo, no se representa la totalidad de la producción en me- dicina clínica, sino sólo la mostrada en una base de datos de ISI Current Contents, que también puede incluir documentos relacionados a otras áreas. Tercero, no se muestra la colaboración entre instituciones, que podría relativizar mejor los índices de producción. Cuarto, sólo empleamos dos indicadores socioeconómicos, elegidos por las facilidades de interpretación. Por último, sólo priorizamos el análisis de un sector de América Latina y el Caribe, pudiendo existir nexos importantes con otros sectores, como algunos países de Centroamérica.

Se recomienda fortalecer o fomentar el desarrollo de sistemas nacionales de investigación de salud, como encargados de la promoción de las actividades en cooperación. Estas actividades deberían promover principalmente la interrelación de investigadores, que formarían las redes y promoverían el recambio generacional. Además, se deben estudiar los programas de colaboración con países no sudamericanos e integrar modelos similares a los programas Sur-Sur. Y en un próximo estudio, se podría analizar la base de datos SCOPUS de forma alternativa, pues permite una visualización más rápida de las colaboraciones, e incluye más revistas sudamericanas, lo que se traduce en un incremento de 1,6 veces el número total de documentos incluidos y en 2,5 veces en el área de medicina, si lo comparamos con ISI-TR.

En conclusión, Brasil y Argentina conservan los mayores valores de producción, colaboración e indicadores de centralidad. Brasil tiene las colaboraciones más intensas con los países sudamericanos, norteamericanos y europeos. A pesar de la heterogeneidad de los indicadores, todos los países han experimentado un incremento en su producción al término del período 2000-2009. Sí existe colaboración entre la mayoría de países de Sudamérica, aunque es proporcional a la producción de cada país, y menor que la colaboración internacional que se da principalmente con EEUU y varios países europeos.

\section{Referencias}

1. Wagner CS, Leydesdorff L. Network structure, self-organization, and the growth of international collaboration in Science. Research Policy 2005; 34: 1608-18.

2. Pellegrini Filho A, Goldbaum M, Silvi J. Producción de artículos científicos sobre salud en seis países de América 
Latina, 1973 a 1992. Rev Panam Salud Pública 1997; 1 (1): 23-34

3. Gupta BM, Dhawan SM. India's collaboration with People's Republic of China in science and technology: a scientometric analysis of coauthored papers during 1994-1999. Scientometrics 2003; 57 (1): 59-74.

4. Peters MA. The rise of global Science and the emerging political economy of international research collaborations. European Journal of Education 2006; 41 (2): 225-44.

5. Chen TJ, Chen YC, Hwang SJ, Chou LF. International collaboration of Clinical Medicine research in Taiwan, 1990-2004: a bibliometric analysis. Journal of the Chinese Medical Association 2007; 70 (3): 110-16.

6. Matthews M, Biglia B, Henadeera K, Desvignes-Hicks JF, Faletic R, Wenholz O (2009). A bibliometric analysis of Australia's international research collaboration in Science and Technology: analytical methods and initial findings. FEAST Discussion Paper 1/09.

7. Programa CYTED. Redes temáticas. [Página en Internet] Hallado en http://www.cyted.org/cyted_informacion/es/redes_tematicas.php. Acceso el 24 de diciembre de 2010

8. Curioso WH, García PJ, Castillo GM, Blas MM, PérezBrumer A, Zimic M, Red QUIPU. Reforzando las Capacidades en Investigación en Informática para la Salud Global en la Región Andina a través de la Colaboración Internacional. Rev Peru Med Exp Salud Pública 2010; 27 (3): 449-57.

9. Alger J, Becerra-Posada F, Kennedy A, Martinelli E, Cuervo LG, Grupo Colaborativo de la Primera Conferencia Latinoamericana de Investigación e Innovación para la Salud. Sistemas nacionales de investigación para la salud en América Latina: una revisión de 14 países. Rev Panam Salud Publica 2009; 26 (5): 447-57.

10. Price DJS. Hacia una Ciencia de la Ciencia. 1973. Barcelona: Ariel.

11. Ríos Gómez C, Herrero Solana V. La producción científica Latinoamericana y la Ciencia mundial: una revisión bibliográfica (1989-2003). Rev Interam Biblio Medellín 2005; 28 (1): 43-61.

12. Fernández MT, Gómez I, Sebastián J. La cooperación científica de los países de América Latina a través de indicadores bibliométricos. Interciencia 1998; 23 (6): 328-37.

13. Sancho R, Morillo F, De Filippo D, Gómez I, Fernández MT. Indicadores de colaboración científica inter-centros en los países de América Latina. Interciencia 2006; 31 (4): 284-92.

14. Sanz Menéndez L. Análisis de Redes Sociales: o como representar las estructuras sociales subyacentes. Apuntes de Ciencia y Tecnología 2003; 7: 21-9.

15. Wren JD, Kozak KZ, Johnson KR, Deakyne SJ, Schilling LM, Dellavalle RP. The write position: a survey of perceived contributions to papers based on byline position and number of authors. EMBO Reports 2007; 8 (11): 988-91.

16. Zbar A, Frank E. Significance of authorships position: an open-ended international assessment. American Journal of the Medical Sciences 2011; 341 (2): 106-9.

17. Mattsson P, Sundberg CJ, Laget P. Is correspondence reflected in the author position? A bibliometric study of the relation between corresponding author and byline position. Scientometrics 2011; 87: 99-105.

18. Costas R, Iribarren-Maestro I. Variations in content and format of ISI databases in their different versions: the case of the Science Citation index in CD-ROM and the web of science. Scientometrics 2007; 72 (2): 167-83.

19. Huamaní C, Mayta-Tristán P. Producción científica peruana en medicina y redes de colaboración, análisis del Science Citation Index 2000-2009. Rev Peru Med Exp Salud Publica 2010; 27 (3): 314-24.

20. Glanzel W. Nacional characteristics in internacional scientific co-authorship relations. Scientometrics. 2001; 15 (1): 69-115.

21. Pereira TS. International dimension of research in Portugal: the European Research Area and beyond. Science and Public Policy 2002; 29 (6): 451-61.

22. Maceira D, Paraje G, Aramayo F, Duarte Masi S, Sánchez D. Financiamiento público de la investigación en salud en cinco países de América Latina. Rev Panam Salud Publica 2010; 27 (6): 442-51.

23. Okubo Y, Zitt M. Searching for research integration across Europe: a closer look at international and interregional collaboration in France. Science and Public Policy 2004; 31 (3): 213-26.

24. Katz JS. Geographical proximity and scientific collaboration. Scientometrics 1994; 31 (1): 31-43.

25. Meneghini R, Packer AL, Nassi-Calò L. Articles by Latin American authors in prestigious journals have fewer citations. Plos One 2008; 3 (11): e3804.

26. Pérez-Padilla R. La inevitable gestación de revistas médicas multinacionales en América Latina. Neumol Cir Torax 2006; 65 (3): 150-4. 